\title{
Effect of Dietary Manipulations (Fasting, Hypocaloric Feeding, and Subsequent Refeeding) on Rat Muscle Energetics as Assessed by Nuclear Magnetic Resonance Spectroscopy
}

\author{
C. Pichard, C. Vaughan, R. Struk, R. L. Armstrong, and K. N. Jeejeebhoy \\ Departments of Medicine, Radiology, and Physics, University of Toronto, Toronto, Ontario, Canada M5S 1A8
}

\begin{abstract}
${ }^{31} \mathrm{P}$ nuclear magnetic resonance (NMR) spectroscopy in vivo and fluorometry were used to measure muscle ATP, total creatine, $\mathrm{pH}$, and $\mathrm{Mg}^{2+}$ in vivo; and to calculate creatine phosphate $(\mathrm{CrP})$, the ratios of $\mathrm{CrP}$ /inorganic phosphate $\left(\mathrm{P}_{i}\right), \mathrm{CrP} /$ ATP, free ADP levels, and the free-energy change in ATP hydrolysis so nutritional effects could be ascertained. These parameters were determined in vivo in resting control, 2-dfasted, and hypocalorically fed rats and in animals similarly hypocalorically fed and then refed. The ATP, $P_{i}$, and intracellular $\mathbf{M g}^{2+}$ levels were comparable in the four groups. When the fasted and underfed animals were compared with the control and refed animals, there were falls in the ratios of $\mathrm{CrP} / \mathrm{P}_{i}$ and $\mathrm{CrP} / \mathrm{ATP}$, in the calculated $\mathrm{CrP}$, and the free-energy change of ATP hydrolysis, but a rise in the calculated free ADP. In the hypocaloric group, intracellular $\mathrm{pH}$ fell significantly and a large peak was noted in the phosphodiester region. The data are consistent with the hypothesis that ATP levels are maintained at the cost of $\mathrm{CrP}$, suggesting that ATP production is disturbed by aerobic and anaerobic mechanisms.
\end{abstract}

\section{Introduction}

Previous studies have shown that malnutrition and hypocaloric feeding in humans and rats resulted in significant changes in the force-frequency curve, relaxation rate, and fatigability of skeletal muscle, together with changes in muscle phosphagens, enzymes, and calcium levels. Refeeding reversed these changes. Analysis of the data led to the formulation of a fourpart hypothesis about the cause of abnormal muscle function due to malnutrition (1).

Central to the proposed hypothesis is a limitation of maximal ATP turnover that accentuates the fall of creatine phosphate $(\mathrm{CrP})^{1}$ during peak contraction. Since the creatine kinase reaction is in equilibrium, this fall must be associated with a rise in free ADP levels and a fall in the free-energy

Address reprint requests to Dr. K. N. Jeejeebhoy, Room 6352, Medical Sciences Building, University of Toronto, Toronto, Ontario M5S 1A8, Canada.

Received for publication 8 September 1987 and in revised form 25 April 1988.

1. Abbreviations used in this paper: ANOVA, analysis of variance; $\mathrm{Cr}$, free creatine; $\mathrm{CrP}$, creatine phosphate; $\Delta \mathrm{G}^{0}$, standard free energy change; $K_{\mathrm{ck}}$, equilibrium constant of the creatine kinase reaction; NMR, nuclear magnetic resonance; $P_{i}$, inorganic phosphate; $Q$, quality factor.

J. Clin. Invest.

(c) The American Society for Clinical Investigation, Inc.

0021-9738/88/09/0895/07 \$2.00

Volume 82, September 1988, 895-901 change of ATP hydrolysis. This in turn would alter calcium pumping and muscle mechanical activity with malnutrition. The concept has been proposed already for exercise-induced fatigue (2). As a prerequisite to testing this hypothesis, it is first necessary to determine muscle ATP, inorganic phosphate $\left(\mathbf{P}_{i}\right)$, free creatine $(\mathrm{Cr}), \mathrm{CrP}, \mathrm{pH}$, and free $\mathrm{Mg}^{2+}$ in vivo and to calculate free ADP levels and the free-energy change in ATP hydrolysis at rest, so that the effects of contraction can then be ascertained. This study thus was undertaken to measure these parameters in vivo, in resting control, 2-d-fasted, and hypocalorically fed rats and in animals similarly hypocalorically fed and then refed.

\section{Methods}

\section{Animals and study protocol}

Male Wistar rats weighing between 230 and $240 \mathrm{~g}$ (7-8 wk old) were obtained for study. Upon arrival from the supplier, the rats were weighed and housed individually in an environmentally controlled atmosphere at an ambient temperature of $22^{\circ} \mathrm{C}$ with a 12-h light-dark cycle. The animals were fed Purina chow (Ralston Purina Co., St. Louis, MO) containing $23 \%$ protein, $4.5 \%$ fat, $51 \%$ carbohydrate, $7.2 \%$ ash, $5.8 \%$ fiber, $2.5 \%$ added minerals, and $6 \%$ water. The animals were assigned to one of four groups: control (18 animals), 2-d fasted (12 animals), $20 \%$ hypocaloric (17 animals) studied after a mean loss of $20 \%$ of their initial weight, and refed ( 7 animals).

The control rats were given food and water freely for $7 \mathrm{~d}$. The 2-d-fasted rats were allowed ad lib. food and water for $5 \mathrm{~d}$ and then received water but no food for $48 \mathrm{~h}$ before study. From the outset, the $20 \%$ hypocaloric group was fed only $5 \mathrm{~g}$ of rat chow daily for a week in parallel with the fed controls. The refed group was given $5 \mathrm{~g}$ of chow daily for a week and then allowed to eat freely for a second week. All groups drank water ad lib. at all times.

\section{Effect of anesthesia on blood $\mathrm{pH}, \mathrm{PCO}_{2}$, and $\mathrm{HCO}_{3}^{-}$}

Aortic blood was taken at the end of the experiment from five rats in each group, and these parameters were measured on a Corning 168 blood gas analyzer (Corning Medical, Corning Glass Works; Medfield, MA).

\section{Nuclear magnetic resonance (NMR) protocol}

The rats were anesthetized with $0.1 / 100 \mathrm{~g}$ body wt i.p. pentobarbital. Then they were individually placed in insulated jackets to preserve body heat while in the chamber (bore of the magnet). Each rat was laid on its side and the right hind leg was inserted in the solenoid coil, which was centered over the bulk of the gastrocnemius muscle. Separate experiments were done to ensure that the animals maintained their body temperature in the insulated covering. The abdomen and other leg were taped back, since it was noted that to obtain good spectral resolution, it was important that no part of the animal rest on the outside of the inductor. In particular, it was necessary to tape up the protruberant abdomens of control and refed rats.

\section{Protocol to determine the source of the spectra}

In a group of eight control animals, the spectra were taken initially as above. Then the skin and subcutaneous tissues were surgically reflected from the underlying muscles and the coil was replaced around the bare 
muscle, centered as before, and the spectra were taken again. To determine directly the contribution of the overlying tissues, the second spectrum (muscle alone) was subtracted from the first spectrum (total, including skin and subcutaneous tissue) by computer.

\section{Solenoid coil}

A solenoid design was selected because the radio frequency field experienced by a sample in such a coil is uniform compared with that experienced by a sample to which a surface coil has been affixed. A coil made of seven turns of polyvinyl chloride-insulated, $50 \%$ flattened, 12-gauge copper wire was wound around a former, $5 \mathrm{~cm}$ long and $2 \mathrm{~cm}$ in diameter. Coil tuning was accomplished using a balanced or floating configuration (3) for the inductor, thereby minimizing the high conductive losses typical of biological materials. The quality factor $(Q)$ of the coil was 120 unloaded, and 60 loaded, at the ${ }^{31} \mathrm{P}$ operating frequency of $34.64 \mathrm{MHz}$. For the standard unbalanced design (4) with the same unloaded $Q$, the loaded $Q$ was 25 . The coil was mounted on a frame constructed so that it was possible to change rats and replace the coil-animal assembly in the bore of the magnet with sufficient accuracy so the shim was not degraded significantly. The initial linewidth was $\sim 15 \mathrm{~Hz}$, and linewidths of $25 \mathrm{~Hz}$ or less were maintained throughout the $10 \mathrm{~h}$ of study in a typical run, during which seven rats were measured in duplicate.

\section{Magnetic field shimming}

Shimming of the static magnetic field was done before each day's run, using the ${ }^{31} \mathrm{P}$ signal from a phosphoric acid phantom that filled the coil. A Lorentzian line $0.520 \mathrm{ppm}$ wide was achieved. The ${ }^{31} \mathrm{P}$ signal from a rat leg was insufficient for shimming, and the $Q$ of the coil was too great to permit the observation of an ${ }^{1} \mathrm{H}$ signal.

\section{Spectral acquisition}

The 100-W driver of the GE 2.0 Tesla spectrometer (General Electric Co., Wilmington, MA) was used to deliver a $90^{\circ}$ pulse in $50 \mu \mathrm{s}$. A pulse of twice this duration yielded a null output, whereas the $50-\mu \mathrm{s}$ pulse yielded a maximum response. The digitizing bandwidth was chosen as $\pm 2 \mathrm{kHz}$ for the phosphorus spectrum $1 \mathrm{kHz}$ wide. Other adjustments were made to minimize hardware-generated phase and baseline distortions.

In the majority of experiments, single pulses were repeated every 4 s, and 128 acquisitions per spectral analysis were accumulated. Four spectra were acquired for each rat.

Each averaged free induction decay signal was baseline corrected, apodized by means of an exponential multiplier, and phased using either the automatic routine or manually. This standard procedure results in an increase of signal to noise at the expense of resolution (3).

To help establish a consistent baseline for calculation of areas under the various peaks, a fitting routine was used to simulate the broad bone curve, which was then subtracted from the spectrum by computer. Relatively, the areas of this broad component were not significantly different in the different groups, since the period of hypocaloric feeding or starvation was too short to reduce bone mass. A check on the validity of the subtraction method was carried out using a two-pulse Hahn echo sequence with a pulse spacing of $125 \mu \mathrm{s}$. The observed echo that occurred $250 \mu \mathrm{s}$ after the first pulse contained a significantly reduced broad component because of its short decay time $(\sim 300 \mu \mathrm{s})$. Both methods gave equivalent results for the $\mathrm{P}_{i}$, ATP, and CrP ratios, indicating that the spin-spin relaxation time $\left(T_{2}\right)$ effects are not important. The exact magnitude of the broad component varied somewhat from rat to rat within each group, depending largely on the position of the leg in the coil. There was no correlation between the deduced ratios of metabolites and the magnitude of the broad component subtracted. A typical spectrum of a control rat without subtraction of the bone curve is given in Fig. 1.

\section{Correction for saturation}

A solution of ATP, $\mathrm{CrP}$, and $\mathrm{P}_{\boldsymbol{i}}$ of the following composition: $7 \mathrm{mM}$ ATP, $10 \mathrm{mM} \mathrm{CrP}, 100 \mathrm{mM} \mathrm{KCl}, 10 \mathrm{mM} \mathrm{NaCl}, 7 \mathrm{mM} \mathrm{MgSO}_{4}$, and 10

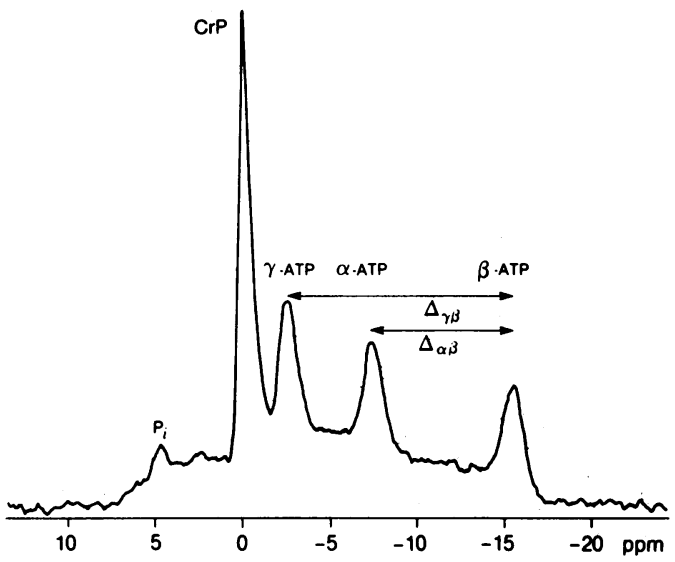

Figure 1. NMR spectrum of control gastrocnemius muscle. $\mathrm{P}_{i}, \mathrm{CrP}$, $\gamma-, \alpha-$, and $\beta$-ATP peaks, and the relative separations between the ATP peaks are identified $\left(\Delta_{\gamma \beta}\right.$ and $\left.\Delta_{\alpha \beta}\right)$. The bone curve has not been subtracted.

$\mathrm{mM} \mathrm{P} \mathrm{P}_{i}$ with the $\mathrm{pH}$ adjusted to 7.15, was used to obtain the correction factors for partial saturation for a repetition time of $4 \mathrm{~s}$. The factors were 1.7 for $\mathrm{P}_{i}, 1.1$ for ATP, and 1.6 for $\mathrm{CrP}$ (Fig. 2). In vivo measurements were carried out to confirm the time constants deduced for the recovery of the CrP and ATP signals. However, the in vivo $\mathbf{P}_{i}$ signal was too weak to use it to provide a reliable time constant for that metabolite.

A repetition rate of $4 \mathrm{~s}$ was chosen, even though this choice required a saturation correction, because of practical considerations, such as the frequency of spectrometer "glitches" and the time interval over which four spectra could be obtained without unduly prolonged observation. Four spectra, which could be compared for consistency and averaged if warranted, were deemed to provide a more reliable result, even though a saturation correction was necessary, than a single spectrum obtained with negligible saturation.

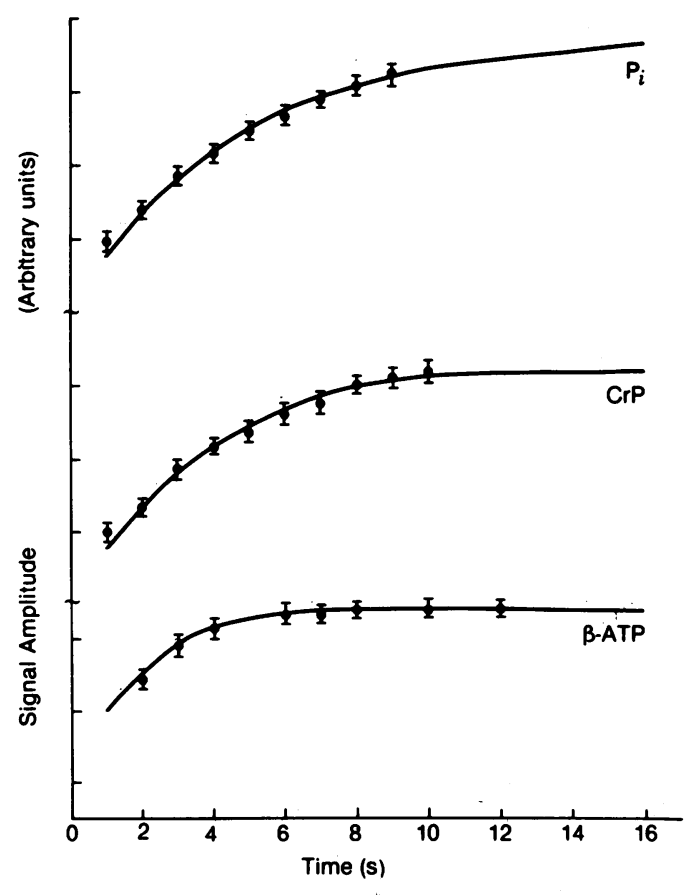

Figure 2. Curves of signal amplitude to repetition time as used to correct for saturation. 


\section{Calibration for $\mathrm{pH}$ measurements}

Solutions of the same composition as above but with a pH range of 6.0 to 7.6 were used to record the shift of the $\mathrm{P}_{i}$ peak with change in $\mathrm{pH}$. Since the $\mathrm{CrP}$ peak does not shift in this $\mathrm{pH}$ range, the degree of separation between the $P_{i}$ and $C r P$ peaks was used to determine the $\mathrm{pH}$. An excellent linear relationship was obtained in the range of biological importance ( $\mathrm{pH}$ 6.7-7.2, data not shown).

\section{Biochemical methods}

After the spectra were obtained, the rat was removed from the chamber, the skin over the leg was dissected away, and the muscle within the coil was rapidly removed and frozen in liquid nitrogen. The rat was then killed. The frozen muscle tissue was lyophilized and biochemical analysis was undertaken for the following metabolites: ATP, CrP, and $\mathrm{Cr}$ by the fluorometric methods of Lowry and Passoneau (5).

\section{Calculations}

Intracellular $p H$. This was obtained by comparing the observed frequency shift between $\mathrm{P}_{i}$ and $\mathrm{CrP}$ with that obtained from the calibration with known $\mathrm{pHs}$.

Ratios of $C r P / P_{i}$ and $C r P / A T P$. As obtained from the spectra, the areas under the $\mathrm{P}_{i}, \mathrm{CrP}$, and beta-ATP peaks were integrated by a computer program and the required ratios were calculated from the respective areas.

Absolute values of $A T P, C r P, C r$, and $P_{i}$. The absolute value of ATP was determined fluorometrically as indicated above. Total creatine was determined by adding together the biochemically determined $\mathrm{CrP}$ and $\mathrm{Cr}$. The values of $\mathrm{CrP}$ and $\mathrm{P}_{i}$ used for the final analysis were calculated from the ratios of $\mathrm{CrP} / \mathrm{ATP}$ and $\mathrm{CrP} / \mathrm{P}_{i}$, using the ATP levels determined fluorometrically. Thus $\mathrm{CrP}=\mathrm{CrP} / \mathrm{ATP} \times \mathrm{ATP}$, and using the $\mathrm{CrP}$ levels so determined, $\mathrm{P}_{i}=1 /\left(\mathrm{CrP} / \mathrm{P}_{i}\right) \times \mathrm{CrP}$. Free creatine was then calculated by subtracting the NMR-calculated $\mathrm{CrP}$ from total creatine.

Free magnesium. Since the relative separations between the beta and alpha, and the beta and gamma peaks of ATP are proportional to the amount of ATP bound to $\mathrm{Mg}^{2+}$, these separations $(\Delta \gamma \beta$ and $\Delta \alpha \beta$ in Fig. 1) were used as an index of the amount of ATP complexed to $\mathrm{Mg}^{2+}$. They were also used to calculate the ratio of free-to-bound $\mathrm{Mg}$ using the relationship noted by Gupta et al. (6). Although there is uncertainty about the exact value of the dissociation constant of MgATP (ATP complexed to magnesium) used to convert this ratio to an absolute value (7) of free $\mathrm{Mg}^{2+}$, nevertheless the results can be used to detect relative changes between the four groups studied. Since the main effect of the free $\mathrm{Mg}^{2+}$ levels is to alter the equilibrium constants of the creatine kinase reaction $\left(K_{\mathrm{ck}}\right)$, the $\Delta \mathrm{G}_{\mathrm{ATP}}^{0}$ (standard free-energy change of ATP hydrolysis), and the $\Delta G_{\text {ATP }}$ (free-energy change of ATP hydrolysis), and also because the published free $\mathrm{Mg}^{2+}$ levels have been reported to be $0.2-0.4(8,9), 1(10)$, and $2.5 \mathrm{mM}(11)$, respectively, we calculated outcomes using this range of values.

The values chosen extended from the low obtained from our calculations (based on the observed separation of the three ATP peaks using the equation of Gupta et al. (6), and a $K_{d}{ }^{\mathrm{MgATP}}$ of $50 \mu \mathrm{M}$ [9]) to the higher values of 1 and $2.5 \mathrm{mM}$ reported in the literature, for our calculations of $K_{\mathrm{ck}}, \Delta \mathrm{G}^{0}{ }_{\mathrm{ATP}}, \Delta \mathrm{G}_{\mathrm{ATP}}$, and free ADP. These ranges were used to determine whether our conclusions about the relative changes between the control and the malnourished and refed groups might be affected by the potential variation in the whole possible range of free $\mathrm{Mg}^{2+}$ levels.

Free ADP. The free ADP levels can be calculated from the assumed equilibrium constant $K_{\text {ck }}$ of the creatine kinase reaction using the following relationship:

$\mathrm{CrP}+\mathrm{ADP}+\mathrm{H}^{+} \rightleftharpoons \mathrm{ATP}+\mathrm{Cr}$

$[\mathrm{ADP}]=[\mathrm{ATP}][\mathrm{Cr}] /[\mathrm{CrP}]\left[\mathrm{H}^{+}\right] K_{\mathrm{ck}}$

The constant $K_{\mathrm{ck}}$ depends (10) upon the $\mathrm{pH}$ and free $\mathrm{Mg}^{2+}$. A range of values therefore was calculated for our purpose, using the equations of
Lawson and Veech (10). Using a computer spread sheet, the values for both the different pHs observed in our study and the assumed free $\mathrm{Mg}^{2+}$ concentrations were then entered into the above equations to obtain a range of values for $K_{\mathrm{ck}}$.

$\Delta G_{A T P}$. The free-energy change of ATP hydrolysis, $\Delta \mathrm{G}_{\mathrm{ATP}}$, in $\mathrm{kJ} /$ mol ATP, can be calculated as follows:

$\Delta \mathrm{G}=\Delta \mathrm{G}^{0}+2.58 \ln [\mathrm{ADP}]\left[\mathrm{P}_{i}\right] /[\mathrm{ATP}]$

However, the absolute $\Delta \mathrm{G}_{\text {ATP }}$ depends upon an estimate of the $\Delta \mathrm{G}^{0}$ that is dependent upon $\mathrm{pH}$, free $\mathrm{Mg}^{2+}$, and temperature. The equations of Alberty (12) were used to calculate a range of $\Delta \mathrm{G}^{0}{ }_{\mathrm{ATP}}$ at a temperature of $37^{\circ} \mathrm{C}$, using the measured $\mathrm{pH}$, together with the range of free $\mathrm{Mg}^{2+}$ levels mentioned above.

The next part of the equation requires the measurement of the free [ADP]/[ATP] ratio, which cannot be done directly from experimental data. However, since the creatine kinase reaction is in equilibrium (13) in rat muscle, the free [ADP] can be derived from Eq. 2 by substituting the right side of Eq. 2 for [ADP] in Eq. 3, yielding

$\Delta \mathrm{G}=\Delta \mathrm{G}^{0}+2.58 \ln [\mathrm{Cr}]\left[\mathrm{P}_{i}\right] /[\mathrm{CrP}]\left[\mathrm{H}^{+}\right] K_{\text {ck }}$

\section{Statistical analysis}

The data are reported as mean $\pm S E$. The overall differences between the four groups were tested by a one-way analysis of variance for statistical significance. If the analysis of variance (ANOVA) was significant, the differences between the control and 2-d-fasted rats, control and hypocalorically fed rats, and control and refed rats, were tested by the $t$ test with the level of significance adjusted for multiple comparisons by Bonferroni's inequality (14). The minimal level of significance for the ANOVA after adjustment for the $t$ tests, was $5 \%$.

\section{Results}

\section{Rat weight}

The mean weight of controls after $7 \mathrm{~d}$ of observation at the time of study rose to $269 \pm 7 \mathrm{~g}$. This was significantly higher than the 2-d-fasted rats weighing $237 \pm 5 \mathrm{~g}$, a difference of $12 \%$. The rats fed hypocalorically for $\sim 1$ wk until they had lost $20 \%$ of their original weight had begun at the same weight as the controls $(238 \pm 5 \mathrm{~g})$ and their weight fell to $179 \pm 3 \mathrm{~g}$ at the time of study. A group of rats similarly fed hypocalorically were then refed for $1 \mathrm{wk}$, during which time they increased in weight to $259 \pm 7 \mathrm{~g}$.

\section{Effect of nutritional manipulations on blood $\mathrm{pH}, \mathrm{PCO}_{2}$, and $\mathrm{HCO}_{3}^{-}$}

The blood pH was normal in controls, 2-d-fasted, 20\% hypocaloric, and refed animals, being 7.42 $\pm 0.02,7.43 \pm 0.02$, $7.40 \pm 0.02$, and $7.43 \pm 0.04$, respectively. The $\mathrm{PCO}_{2}$ was similarly normal, being $42.9 \pm 4.5,36.4 \pm 3.7,44.4 \pm 2.1$, and $41.9 \pm 5.6 \mathrm{mmHg}$, respectively. The $\mathrm{HCO}_{3}^{-}$was $28.1 \pm 1.6$, $23.9 \pm 0.7,27.1 \pm 0.2$, and $27.4 \pm 1.8 \mathrm{mM}$, respectively. None of the differences was significant by ANOVA. There thus was no evidence of blood acidosis that could have influenced the results.

\section{Qualitative changes in NMR spectra between controls, 2-d-fasted, and hypocalorically fed animals}

The data are illustrated in Figs. 1 and 3. Note that in Fig. $3 B$, the size of the CrP peak is decreased in relation to the others and a prominent peak has appeared in the region that corresponds to phosphodiesters (15). 

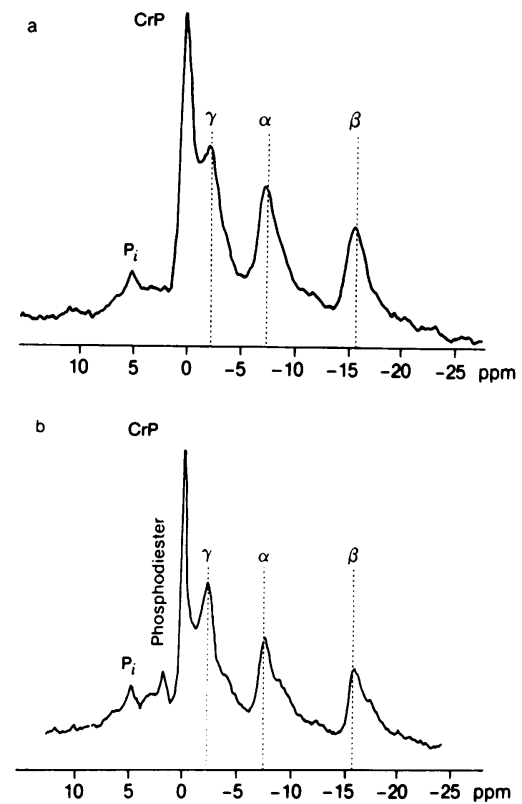

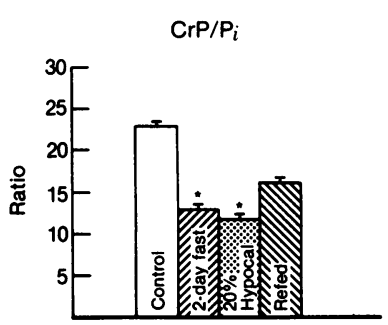

Figure 5. The ratios of $\mathrm{CrP}$ to $\mathrm{P}_{i}$ for all groups. ${ }^{*} P<0.01$.
Figure 3. NMR spectra of the gastrocnemius muscle of $(A)$ 2-d-fasted and $(B)$ hypocalorically fed rats. The CrP peak is relatively decreased, and a prominent peak has appeared in the phosphodiester region $(B)$.

\section{Contribution of skin, hair, and subcutaneous tissue to the spectra}

Subtraction of spectra taken with the coil surrounding the muscle from the spectra taken over the intact leg resulted in only a baseline without peaks. There was thus no significant contribution of the skin, fat, and subcutaneous tissue to the spectra obtained in this study.

\section{Ratio of CrP to ATP}

The ratio of CrP/ATP in control animals is noted in Fig. 4. There was a highly significant difference by ANOVA ( $F$ $=25.3$, degrees of freedom (df) 3 and 50; $P<0.01$ ). Individually, the control ratio was significantly higher than that of the 2-d-fasted and the hypocalorically fed rats (adjusted $P<0.001$ for both), but not different from that of the refed animals.

\section{Ratio of CrP to $P_{i}$}

The data are shown in Fig. 5. There was a highly significant difference by ANOVA $(F=12.97$, df 3 and $50 ; P<0.01)$. The ratio for control rats was found to be significantly higher than for the 2-d-fasted and hypocalorically fed rats $(P<0.001)$ but not for the refed animals.

\section{Muscle $p H$}

The data are illustrated in Fig. 6. There was a significant difference by ANOVA $(F=3.22$, df 3 and $50 ; P<0.05)$. The $\mathrm{pH}$ of the $20 \%$ hypocaloric group, but not of the 2-d-fasted and refed groups, was significantly but minimally lower than controls, the means being $7.12 \pm 0.01$ and $7.15 \pm 0.01$, respectively $(P<0.01)$.

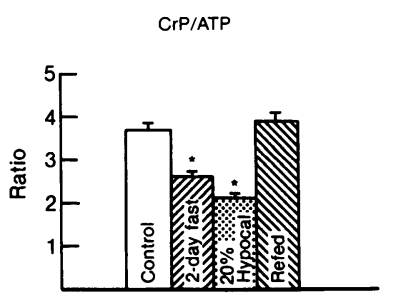

Figure 4. The ratios of $\mathrm{CrP}$ to ATP for all groups. ${ }^{*} P<0.01$.

\section{Muscle free $\mathrm{Mg}^{2+}$}

This was estimated indirectly from the separation of the alpha and beta peaks of the ATP spectrum, this distance being independent of $\mathrm{pH}$ changes (6). There was no significant difference by ANOVA $(F=0.52$, df 3 and 46$)$.

A more exact estimate of the free-to-bound $\left(\mathrm{Mg}^{2+}\right.$ to MgATP) was obtained by the equation of Gupta et al. (6). These ratios were $0.105 \pm 0.008,0.107 \pm 0.011,0.101 \pm 0.012$, and $0.085 \pm 0.013$ for control, 2-d-fasted, $20 \%$ hypocaloric, and refed groups, respectively. ( $F=0.52$, df 3 and $46 ; P=\mathrm{NS}$ ). When the free $\mathrm{Mg}^{2+}$ was calculated from these ratios using the relationship given by Gupta et al. (9) and their apparent dissociation constant $K_{d}{ }^{\text {MgATP }}$ of $50 \mu \mathrm{M}$ (9), the free $\mathrm{Mg}^{2+}$ values were $532 \pm 97,474 \pm 60,517 \pm 71$, and $628 \pm 105 \mu \mathrm{M}(F=0.74$, df 3 and $46 ; P=\mathrm{NS}$ ).

\section{Effect of $\mathrm{pH}$ and $\mathrm{Mg}^{2+}$ differences on the equilibrium constant of the creatine kinase reaction $\left(K_{c k}\right)$ and the $\Delta G^{0}$ in $A T P$ hydrolysis}

The differences in $\mathrm{pH}$ and $\mathrm{Mg}^{2+}$ between the groups were small in magnitude and only statistically significant for $\mathrm{pH}$. They had little effect on the calculated $K_{\text {ck }}$ and the $\Delta G^{0}{ }_{\text {ATP }}$.

Our study's calculated data are tabled (Table I) for comparison with the values obtained when the calculations are based on an assumed free $\mathrm{Mg}^{2+}$ of $1 \mathrm{mM}$, as used by Lawson and Veech (10), and the higher one of $2.5 \mathrm{mM}$ obtained by Wu et al. (11). It can be seen that although the level of free $\mathbf{M g}^{2+}$ clearly alters the $K_{\mathrm{ck}}$ and $\Delta \mathrm{G}_{\mathrm{ATP}}^{0}$, the relative differences between the groups due to $\mathrm{pH}$ variation is small. As shown below, when these various values for free $\mathbf{M g}^{2+}$ are used to calculate the free ADP levels and $\Delta \mathrm{G}_{\mathrm{ATP}}$ of the experimental groups, they do not change the significance of the differences observed between them.

\section{Muscle ATP, CrP, Cr, total creatine, and $P_{i}$ levels}

Muscle ATP. The data are given in Fig. 7. There was no significant difference in the ATP content by ANOVA $(F=0.16$, df 3 and 50).

Muscle total creatine. There was a significant difference by ANOVA $(F=8.84$, df 3 and $45 ; P<0.01)$. The levels were

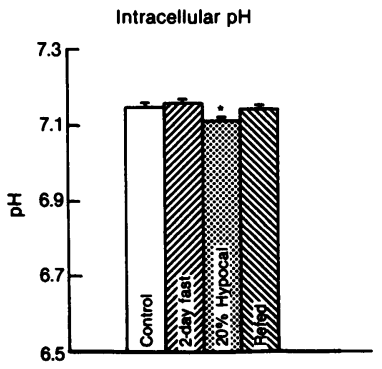

Figure 6. Intracellular $\mathrm{pH}$ for all groups. ${ }^{*} P<0.01$. 
Table I. Effect of $\mathrm{pH}$ and Free $\mathrm{Mg}^{2+}$ Differences on the Equilibrium Constant of the Creatine Kinase Reaction and $\Delta G^{0}$ of $A T P$ Hydrolysis

\begin{tabular}{cccc}
\hline Free $\mathrm{Mg}^{2+}$ & $\mathrm{pH}$ & $K_{\text {ck }}$ & $\Delta \mathrm{G}^{0}{ }_{\text {ATP }}$ \\
\hline$m M$ & & $\times 10^{9}$ & $k J / m o l$ \\
0.097 & 7.15 & 0.56 & 38.55 \\
0.097 & 7.12 & 0.56 & 38.46 \\
0.53 & 7.15 & 1.24 & 36.54 \\
0.53 & 7.12 & 1.24 & 36.45 \\
1.00 & 7.15 & 1.68 & 36.11 \\
1.00 & 7.12 & 1.68 & 35.97 \\
2.50 & 7.15 & 2.33 & 35.95 \\
2.50 & 7.12 & 2.32 & 35.79 \\
\hline
\end{tabular}

$47.5 \pm 3.0,35.9 \pm 1.7,43.9 \pm 1.6$, and $51.3 \pm 4.4 \mathrm{mM} / \mathrm{g}$ wet weight in controls, 2-d-fasted, $20 \%$ hypocaloric, and refed animals, respectively. The only individual difference that was significant was that between control and 2-d-fasted rats $(P<0.001)$. Acute but not chronic malnutrition thus reduced muscle total creatine in relation to total weight.

Creatine phosphate and free creatine levels. The data for creatine phosphate in muscle can be seen in Fig. 7. There was a highly significant difference by ANOVA $(F=23.05$, df 3 and $50 ; P<0.01)$. Individually, control values were significantly higher than those for 2-d-fasted $(P<0.001)$ and for hypocalorically fed animals $(P<0.001)$, but not different from those for refed animals.

In comparison, free creatine levels rose in hypocalorically fed animals when compared with controls (Fig. 7, $F=2.31$; df 3 and $46 ; P=N S$ ), but just failed to attain statistical signifcance.

Free phosphorus levels. The data in Fig. 7 show no significant difference between groups by ANOVA $(F=1.4$, df 3 and 50).

\section{Free ADP levels}

The calculated free ADP levels are given in Fig. 8. There was a significant difference by ANOVA $(P<0.01)$ irrespective of the free $\mathrm{Mg}^{2+}$ level assumed. Individually, only the hypocalorically fed animals showed a significant difference (of elevation) among the four groups $(P<0.001)$.

\section{Free-energy change for ATP hydrolysis}

The data are set out in Fig. 9. There was a significant difference by ANOVA $(P<0.01)$ irrespective of the assumed free $\mathrm{Mg}^{2+}$

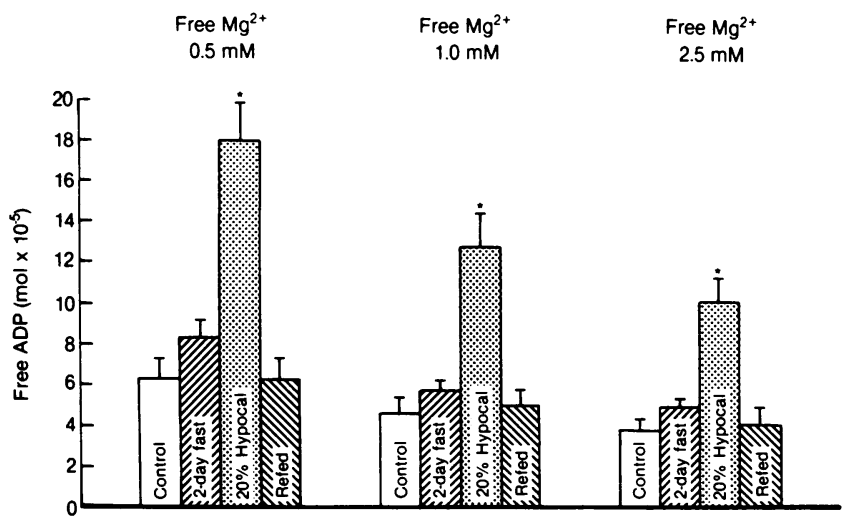

Figure 8. Levels of calculated free ADP for all groups. Sets of values for each of the three assumed concentrations of free $\mathrm{Mg}^{2+}$ are shown. ${ }^{*} P<0.01$.

level. Individually, the free-energy change for ATP hydrolysis was significantly lower in the 2-d-fasted and hypocalorically fed animals than in the controls $(P<0.01)$.

\section{Phosphodiester levels}

The phosphodiester peak was representative of $0.78 \pm 0.32$, $1.44 \pm 0.27,1.78 \pm 0.24$, and $0.70 \pm 0.10 \mathrm{mM} / \mathrm{kg}$ wet weight in controls, 2-d-fasted, $20 \%$ hypocaloric, and refed animals, respectively. There was a significant difference by ANOVA ( $F$ $=3.28$; df 3 and $43 ; P<0.05$ ). Individually, only in the hypocaloric group was the amount significantly greater than in the controls $(P<0.05)$.

\section{Discussion}

Control and refed animals. Levels of ATP, $\mathrm{CrP}, \mathrm{P}_{i}$, and $\mathrm{pH}$ of control animals are similar to ones published for cat biceps, a comparable fast-twitch muscle (16). In controls the following mean ratios were determined. $\mathrm{CrP} /$ total phosphorus (ATP $+\mathrm{CrP}+\mathrm{P}_{i}$ ) was $0.758, \mathrm{P}_{i} /$ total phosphorus was 0.039 , and ATP/total phosphorus was 0.202 , values almost identical to those published by Meyer et al. (16). The calculated free ADP levels are in the range published by Veech et al. (17) if the free $\mathrm{Mg}^{2+}$ assumed is $1 \mathrm{mM}$. Our $\Delta \mathrm{G}_{\mathrm{ATP}}$ for muscle of $-68 \mathrm{~kJ} / \mathrm{mol}$ is similar to the $-72 \mathrm{~kJ} / \mathrm{mol}$ reported for cat biceps (16). Animals refed after hypocaloric feeding showed no difference from controls.

Effect of a 2-d-fast and hypocaloric feeding. The striking effect was a significant fall in $\mathrm{CrP}$, which was associated with a loss of muscle total creatine in the 2-d-fasted rat. The reserves of energy phosphorus thus decreased, whereas the calculated

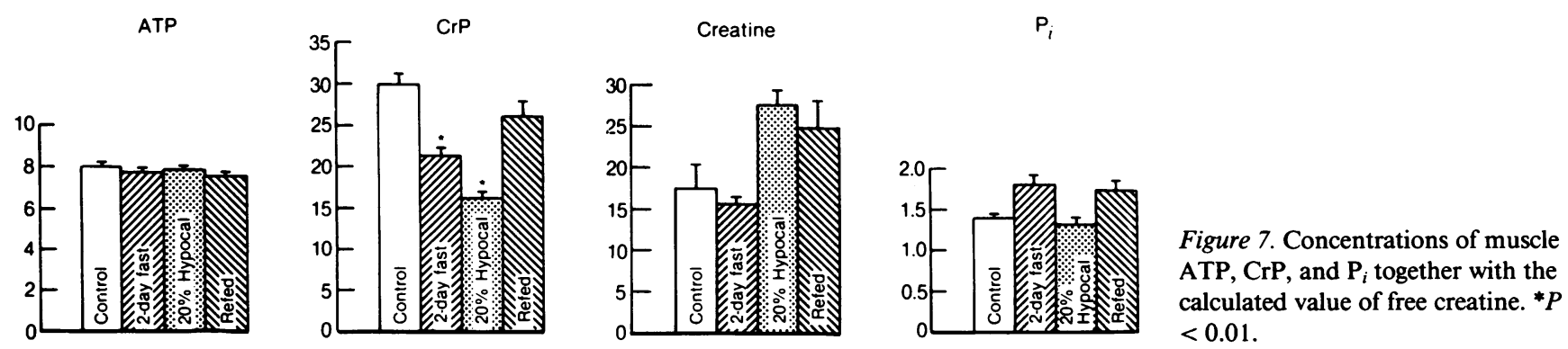




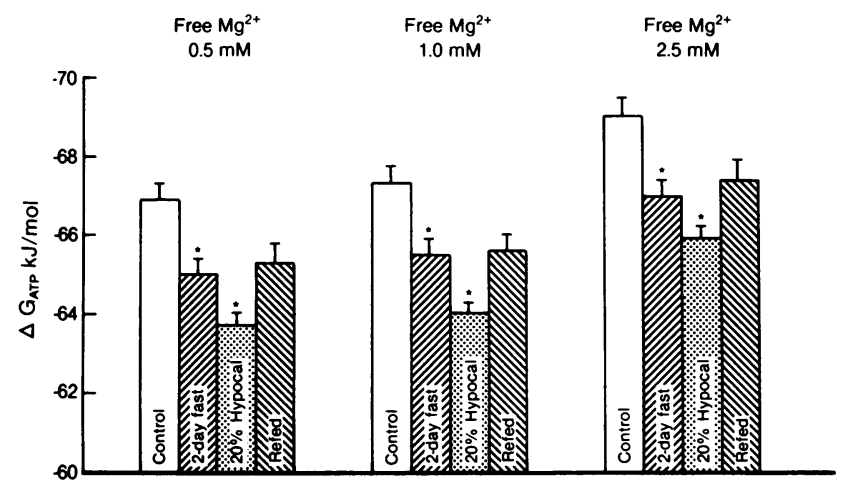

Figure 9. Free-energy change for ATP hydrolysis. Sets of values for each of the three assumed concentrations of free $\mathrm{Mg}^{2+}$ are shown. ${ }^{*} P$ $<0.01$.

$\Delta \mathrm{G}_{\mathrm{ATP}}$ fell significantly. It is of interest that Dawson et al. (2) found that in fatigued muscle, a fall in $\Delta \mathrm{G}_{\mathrm{ATP}}$ was related to a slower relaxation rate, in such a manner that small changes in $\Delta \mathrm{G}_{\mathrm{ATP}}$ were associated with significant changes in relaxation rates.

This effect of change in the $\Delta G_{A T P}$ on relaxation rate was hypothesized to be due to slower pumping of calcium (2), a concept supported by Kodoma (18). We (19) and others (20) have also observed that fasted and hypocalorically fed rats and malnourished humans have a slower relaxation rate, perhaps, as shown herein, due to a fall in $\Delta \mathbf{G}_{\mathrm{ATP}}$.

Effect of $p H$ and free $M g^{2+}$ levels on free $A D P$ and $\Delta G_{A T P}$. It is recognized that the above factors will alter the $K_{\mathrm{ck}}$ and the $\Delta \mathrm{G}_{\mathrm{ATP}}$. However, the differences in $\mathrm{pH}$ were small and the actual values influenced the calculation to a minor extent (Table I). The free $\mathrm{Mg}^{2+}$ levels obtained by us from the NMR data are consistent with the recent observations of Gupta et al. (9) and Maughan (8). Despite the uncertainty of the precise free $\mathrm{Mg}^{2+}$ level, it is clear that the differences between the groups for $\mathrm{CrP}$, free $\mathrm{ADP}$, and $\Delta \mathrm{G}_{\mathrm{ATP}}$ remain statistically significant.

Changes in phosphodiester-like substances. An obvious peak appears between the $\mathrm{P}_{i}$ and $\mathrm{CrP}$ in the hypocalorically fed animal. This peak is in the region of phosphodiester compounds and of inosine monophosphate (IMP) (16). Although a rise in IMP is usually seen after vigorous exercise (17), it is known that small changes in the ATP/ADP ratio cause much larger changes in AMP, and thus activate AMP deaminase (21), leading to an increase in IMP (21).

Significance of the observed changes. The changes are not due to acidosis or to anoxia of the muscle because the blood $\mathrm{pH}, \mathrm{PCO}_{2}$, and $\mathrm{HCO}_{3}^{-}$were normal and not different between the groups. Furthermore, muscle $\mathrm{pH}$ was no different in the 2-d-fasted animals, which showed a marked fall in $\mathrm{CrP}$ and $\Delta \mathrm{G}_{\mathrm{ATP}}$ and a rise in free ADP. Even in hypocaloric animals, the muscle $\mathrm{pH}$ fell by only 0.03 . In preliminary studies in starved rats, Jacobs et al. (22) concluded that intracellular ATP was maintained at the expense of $\mathrm{CrP}$.

Muscle biopsy data in critically ill septic subjects have shown lower phosphagens (23). These findings are similar to those related to hypocalorically fed rats in this study. The fall in CrP and rise in free ADP have been noted in fatigue (2). However, there are major differences. In fatigue there is a significant fall in $\mathrm{pH}($ to $<7.0)$ and a rise in $\mathbf{P}_{i}$; total creatine remains constant except in severe exercise (24). In malnutrition, the $\mathrm{P}_{i}$ was not significantly different from that in controls; therefore changes in $\mathrm{P}_{i}$ concentration could not cause the change in the force-frequency curve and relaxation rate seen in the gastrocnemius of malnourished rats (19). The fall in $\mathrm{CrP}$ and rise in free ADP could be due to a change in fiber type (25). NMR studies in cat muscle have shown that fast-twitch fibers have higher ATP, lower free ADP and CrP, and markedly lower $P_{i}$ levels compared with slow-twitch fibers (17). Since malnutrition was not associated with a rise in $P_{i}$, nor in a significant fall in ATP, an alteration in fiber type cannot entirely explain the observed findings. The changes are more likely due to a loss of muscle $\mathrm{CrP}$ relative to total creatine, resulting in a relative loss of energy reserve. Since ADP is rapidly translocated into the mitochondria and phosphorylated to ATP, a rise in the ADP could result from deficient oxidative phosphorylation. The findings are consistent with the hypothesis that the reduced phosphofructokinase and succinic dehydrogenase levels observed in malnutrition $(25,26)$ may limit the flux of glycolytic and oxidative pathways. A recent study of the effect of 5-d fasting on the ${ }^{31} \mathrm{P}$ NMR of the human forearm (27) has shown reduced lactate production and changes in the $\mathrm{P}_{i} / \mathrm{CrP}$ ratio consistent with a limitation of anaerobic glycolysis. These findings, together with ours, support the proposed hypotheses (1).

\section{Acknowledgments}

This work was supported by Medical Research Council of Canada grant MA.9814. Dr. Pichard received training awards from the Janggen-Pöhn Stiftung, St.-Gallen, Switzerland and from the Société Académique Vaudoise, Lausanne, Switzerland.

\section{References}

1. Jeejeebhoy, K. N. 1986. Muscle function and nutrition. Gut. 27(Suppl. 1):25-39.

2. Dawson, M. J., D. C. Gadian, and D. R. Wilkie. 1980. Mechanical relaxation rate and metabolism studied in fatiguing muscle by phosphorus nuclear magnetic resonance. J. Physiol. (Lond.). 299:465-484.

3. Fukushima, E., and S. B. W. Roeder. 1981. Experimental Pulse NMR: A Nuts and Bolts Approach. Addison-Wesley Publishing Co. Inc. Reading, MA. 414.

4. The Radio Amateur's Handbook. 1986. American Radio Relay League Inc., Newington, CT. Section 3, p. 12; section 16, p. 6.

5. Lowry, O. H., and J. V. Passoneau. 1972. A collection of metabolite assays. In A Flexible System of Enzymatic Analysis. Academic Press, New York. 146-160.

6. Gupta, R. K., and R. D. Moore. 1980. ${ }^{31}$ P NMR studies of intracellular free $\mathrm{Mg}^{2+}$ in intact frog skeletal muscle. J. Biol. Chem. 255:3987-3993.

7. Meyer, R. A., M. J. Kushmerick, and T. R. Brown. 1982. Application of ${ }^{31} \mathrm{P}-\mathrm{NMR}$ spectroscopy to the study of striated muscle metabolism. Am. J. Physiol. 242.(Cell Physiol. 11):C1-C11.

8. Maughan, D. 1983. Diffusible magnesium in frog skeletal muscle cells. Biophys. J. 43:75-80.

9. Gupta, R. K., P. Gupta, W. D. Yushok, and Z. B. Rose. 1983. Measurement of the dissociation constant of MgATP at physiological nucleotide levels by a combination of ${ }^{31} \mathrm{P}$ NMR and optical absorbance spectroscopy. Biochem. Biophys. Res. Commun. 117:210-216.

10. Lawson, J. W. R., and R. L. Veech. 1979. Effects of pH and free $\mathrm{Mg}^{2+}$ on the $\mathrm{K}_{\mathrm{eq}}$ of the creatine kinase reaction and other phosphate hydrolyses and phosphate transfer reactions. J. Biol. Chem. 254:6528-6537. 
11. Wu, S. T., G. M. Pieper, J. M. Salhany, and R. S. Eliot. 1981. Measurement of free magnesium in perfused ischemic arrested heart muscle. A quantitative phosphorus-31 nuclear magnetic resonance and multiequilibria analysis. Biochemistry. 20:7399-7403.

12. Alberty, R. A. 1972. Calculation of the standard Gibbs free energy, enthalpy and entropy changes for the hydrolysis of ATP at $0^{\circ}$, $25^{\circ}, 37^{\circ}$, and $75^{\circ}$. In Horizons of Bioenergetics. A. San Pietro and $\mathrm{H}$. Gest, editors. Academic Press, New York. 135-147.

13. Shoubridge, E. A., J. L. Bland, and G. K. Radda. 1984. Regulation of creatine kinase during steady-state isometric twitch contraction in rat skeletal muscle. Biochim. Biophys. Acta. 805:72-78.

14. Ware, J. H., F. Mosteller, and J. A. Ingelfinger. 1986. $P$ values. In Medical Uses of Statistics. J. C. Bailar III and F. Mosteller, editors. Massachusetts Medical Society (NEJM Books), Waltham, MA. 149-169.

15. Williams, S. R., and D. G. Gadian. 1986. Tissue metabolism studied in vivo by nuclear magnetic resonance. Q. J. Exp. Physiol. 71:335-360.

16. Meyer, R. A., T. R. Brown, and M. J. Kushmerick. 1985. Phosphorus nuclear magnetic resonance of fast- and slow-twitch muscle. Am. J. Physiol. 248:(Cell Physiol. 17):C279-C287.

17. Veech, R. L., J. W. R. Lawson, N. W. Cornell, and H. A. Krebs. 1979. Cytosolic phosphorylation potential. J. Biol. Chem. 254:65386547.

18. Kodama, T. 1985. Thermodynamic analysis of muscle ATPase mechanisms. Physiol. Rev. 65:468-551.

19. Russell, D. McR., H. L. Atwood, J. S. Whittaker, T. Itakura, P. M. Walker, D. A. G. Mickle, and K. N. Jeejeebhoy. 1984. The effect of fasting and hypocaloric diets on the functional and metabolic characteristics of rat gastrocnemius muscle. Clin. Sci. 67:185-194.

20. Chan, S. T. F., S. J. McLaughlin, G. A. Ponting, J. Biglin, and H. A. Dudley. 1986. Muscle power after glucose-potassium loading in undernourished patients. Br. Med. J. 293:1055-1056.

21. Newsholme, E. A., and A. R. Veech. 1983. Biochemistry for the Medical Sciences. John Wiley \& Sons, Ltd., Chichester, England. 315-316.

22. Jacobs, D. O., G. Whitman, J. Maris, R. Settle, M. J. Kolda, A. H. Harken, and J. Rombeau. 1985. In vivo $\mathrm{P} 31$ nuclear magnetic resonance spectroscopy of rat skeletal muscle during starvation. $J$. Parenter. Enteral Nutr. 9:107. (Abstr.)

23. Liaw, K. Y., J. Askanazi, C. B. Michelson, L. R. Kantrowitz, P. Furst, and J. M. Kinney. 1980. Effect of injury and sepsis on high-energy phosphate in muscle and red cells. J. Trauma. 20:755-759.

24. Sahlin, K., G. Palmskog, and E. Hultman. 1978. Adenine nucleotide and IMP contents of the quadriceps muscle in man after exercise. Pfluegers Arch. Eur. J. Physiol. 374:193-198.

25. Russell, D. McR., P. M. Walker, L. A. Leiter, A. A. F. Sima, W. K. Tanner, D. A. G. Mickle, J. Whitwell, E. B. Marliss, and K. N. Jeejeebhoy. 1984. Metabolic and structural changes in skeletal muscle during hypocaloric dieting. Am. J. Clin. Nutr. 39:503-513.

26. Church, J. M., B. Y. Choong, and G. L. Hill. 1986. Abnormal muscle fructose bisphosphatase activity in malnourished cancer patients. Cancer. 58:2448-2452.

27. Lunt, J. A., P. S. Allen, M. Brauer, D. Swinamer, E. O. Treiber, A. Belcastro, R. Eccles, and E. G. King. 1986. An evaluation of the effect of fasting on the exercise-induced changes in $\mathrm{pH}$ and $\mathrm{P}_{i} / \mathrm{PCr}$ from skeletal muscle. Magn. Reson. Med. 3:946-952. 